\title{
Synthesis of iron phosphate-SAPO-34 composite and its application as effective absorbent for wastewater treatment
}

\author{
Ying Cao ${ }^{1,2}$, Lianmei Wei ${ }^{1}$, Xianzhen Song ${ }^{1}$, Xixi Yan ${ }^{1}$, Xiaoyu Liu ${ }^{1}$ and Lijun Wang ${ }^{1,2, a}$ \\ ${ }^{1}$ Facultyof Environmental and Materials Engineering, Shanghai Polytechnic University, Shanghai 201209, P.R. China \\ ${ }^{2}$ Research Center of Resource Recycling Science and Engineering, Shanghai Polytechnic University, Shanghai 201209, P.R. China
}

\begin{abstract}
High-purity $\mathrm{FePO}_{4}$ was purified from iron-based phosphating slag as raw material, and $\mathrm{FePO}_{4} @$ SAPO-34 was synthesized by hydrothermal crystallization method under the action of templating agent-diethylamine. The synth esized $\mathrm{FePO}_{4} @$ SAPO-34 samples were characterized by x-ray diffraction (XRD), scanning electron microscopy (SE M) and Fourier transform infrared spectroscopy (FT-IR). The effects of different crystallization time on the morpholo gy and crystallization of $\mathrm{FePO}_{4} @$ SAPO-34 crystals were investigated. The removal of heavy metal ion wastewater by low-cost $\mathrm{FePO}_{4} @ \mathrm{SAPO}-34$ was investigated. The experimental results show that when the reaction time is $180{ }^{\circ} \mathrm{C}$ an $\mathrm{d}$ the reaction time is $72 \mathrm{~h}$, the crystallization of $\mathrm{FePO}_{4} @ \mathrm{SAPO}-34$ is the best. When the dosage is $0.6 \mathrm{~g}$, the removal e fficiency of heavy metal ions is the highest.
\end{abstract}

\section{Introduction}

Phosphating is a commonly used surface treatment technology. Phosphating slag is a precipitated substance that is produced on the surface of a metal phosphating process. Its main components are metal ions such as phosphate, iron and zinc [1]. In order to effectively control the pollution of phosphating slag, researchers have carried out a lot of research work and made some progress. In this study, the iron phosphate is purified by phosphating slag and has various crystal structures. It is provided with unique catalytic properties, ion exchange properties, etc. Research reports show that iron phosphate is negatively charged (zeta $=-30 \sim-40 \mathrm{mV}$ ) in wastewater, and Its electronegative properties produce electrostatic adsorption on positively charged ions [2].

The molecular sieve has a uniform pore structure. According to the molecular size, it can selectively adsorb. Thus, it is mostly used for adsorbents and catalysts [3]. In 1982, SAPO-34 was first synthesized as a SAPOS series molecular sieve by Wilson et al [4]. It has CHA structure and medium strength acid center. SAPO-34 exhibits excellent performance in adsorption and catalysis [5]. Because SAPO-34 has a neutral framework structure, it is difficult to provide the active center required for many reactions [6]. Therefore, it is very important to modify the material. In summary, this study prepared a new catalytic adsorption material of $\mathrm{FePO}_{4} @$ SAPO-34. New materials were applied to remove heavy metal ions from wastewater.

\section{Materials and methods}

\subsection{Materials}

Phosphate residue was supplied by an auto components company in Zhejiang, China. $\mathrm{Pb}\left(\mathrm{NO}_{3}\right)_{2}$ (analytical regent $(A R), \geqslant 99.9 \%)$, pseudo boehmite $\left(\mathrm{Al}_{2} \mathrm{O}_{3}, \geqslant 67.5 \%\right)$, phosphoric acid $(\mathrm{AR}, \geqslant 85 \%)$ and diethylamine (AR, 99\%) were obtained from Sinopharm Chemical Reagent Co. Ltd., of Shanghai, China. Silica solution $\left(\mathrm{SiO}_{2}, \geqslant 30 \%\right)$ was purchased from Qingdao Haiyang Chemical Co.,Ltd[4]. Other chemicals used in the present work were of ananlytical grade and used without further purification. Deionized water was produced by an ultrapure water system (UPT-I-60L, Super Pure Technology Corporation, China).

\subsection{Adsorbent preparation}

A certain amount of phosphating slag, water and phosphoric acid were uniformly mixed in an appropriate ratio, and placed in an oven at $80^{\circ} \mathrm{C}$ for $4 \mathrm{~h}$, and the sample was suction filtered and dried overnight; after three times of reaction, a phosphating slag was obtained to purify the sample iron phosphate. The experiment used hydrothermal synthesis of $\mathrm{FePO}_{4} @$ SAPO-34. Firstly, the silicon source, the phosphorus source, the aluminum source, the $\mathrm{FePO}_{4}$ and the appropriate amount of water are uniformly mixed according to a certain ratio, and then

\footnotetext{
a Corresponding author: ljwang@ @spu.edu.cn
} 
the templating agent diethylamine is added, and after pretreatment, it is transferred to a polytetrafluoroethylene reactor, and heated to $180{ }^{\circ} \mathrm{C}$, and is self-generated[5]. Crystallize under pressure for different times, after suction filtration, washing and drying. Then, it was baked in a muffle furnace at $550{ }^{\circ} \mathrm{C}$ for $6 \mathrm{~h}$ to obtain a modified new material.

\subsection{Heavy metal ion removal experiment}

The experiment of removal of heavy metal ions in simulated wastewater was carried out with a material dosage of $0.6 \mathrm{~g}$, lead-containing $\left(\mathrm{Pb}\left(\mathrm{NO}_{3}\right)_{2}\right)$ wastewater concentration of $100 \mathrm{mg} / \mathrm{L}$. Adesignated intervals, samples of the solution were collected and immediately filter to monitor the adsorption efficiency by inductively coupled plasma-atomic emission spectrometry (ICP-AES) (ICAP 6000, Thermo Corporation, USA). All adsorption experiments were stirred at $350 \mathrm{r} / \mathrm{min}$. After adsorption experiment, the FePO4@SAPO-34 adsorbent was collected with strong acid and then reused.

\subsection{Characterization methods}

The morphology of the samples was characterized by sca-nning electron microscopy (SEM) (S-4800, Hitachi Corp-oration,Japan) with the accelerating voltage at 10 $\mathrm{kV}$ and current at $10 \mathrm{~mA}$. X-ray diffraction (XRD) (D8Advance, Bruker Corporation, Germany) measure ments were un-dertaken to record the phase composition and crystalline structure of the powder samples, operating at $40 \mathrm{kV}$ and $40 \mathrm{~mA}$ with a monochromatized $\mathrm{Cu} \mathrm{K} \alpha$ radiation $(\lambda=0.15418 \mathrm{~nm})$ source. The chemical bonds on the surface of the samples were examined by Fourier transform infrared spectroscopy (FTIR) (Vertex70, Bruker Corporation, Germany). $\mathrm{KBr}$ was chosen as a diluent. The surface area was measured based on the Brunauer Emmett Teller (BET) (ASAP-2020M, Micromeritics Corporation, USA) model over a relative pressure range of $0.05-1$.

\section{Results and discussion}

\subsection{XRD characterization}

Figure 1 is an XRD pattern of a sample synthesized at different crystallization times. Crystallization plays a role in transforming metastable materials into stable crystals. So crystallization time is one of the most important factors that affect the synthesis of zeolite. Fig1. shows five peaks at $9.48^{\circ}, 16.2^{\circ}, 20.7^{\circ}, 25^{\circ}$ and $31^{\circ}$, corresponding to (101), (211), (104), (401) of SAPO-34
(JCPDS), respectively [6-7]. With the extension of time, the peak intensity gradually increased. When $t=72 \mathrm{~h}$, the peak intensity was the strongest, but when the time was extended to $84 \mathrm{~h}$, the peak intensity weakened and the impurity peak began to appear. The crystallinity of SAPO-34 was set to $100 \%$. The relative crystallinity of FePO4@SAPO-34 is calculated as follows [7-8]:

$$
X_{i}(\%)=X_{s} \frac{\sum I_{i}}{\sum I_{s}}
$$

Where, $\mathrm{X}_{\mathrm{i}}$ is relative crystallinity of the sample; $\mathrm{Xs}_{\mathrm{s}}$ is equivalent to $100 \% ; \sum \mathrm{I}_{\mathrm{i}}$ is the sum of the five characteristic peak intensities of the sample; $\sum I_{S}$ is the sum of the five characteristic peak intensities of the standard sample.

From Fig. 3, the relative crystallinity of FePO4@SAPO-34 increased from $40 \%$ to 98\%, indicating that the crystallinity of the sample increased with the crystallization time. But when the time is too long, it could lead to the formation of other crystals. Too short or too long crystallization time was not conducive to the synthesis of FePO4@SAPO-34. The crystallization time was the most favourable for the synthesized FePO4@SAPO-34 at 72 h.

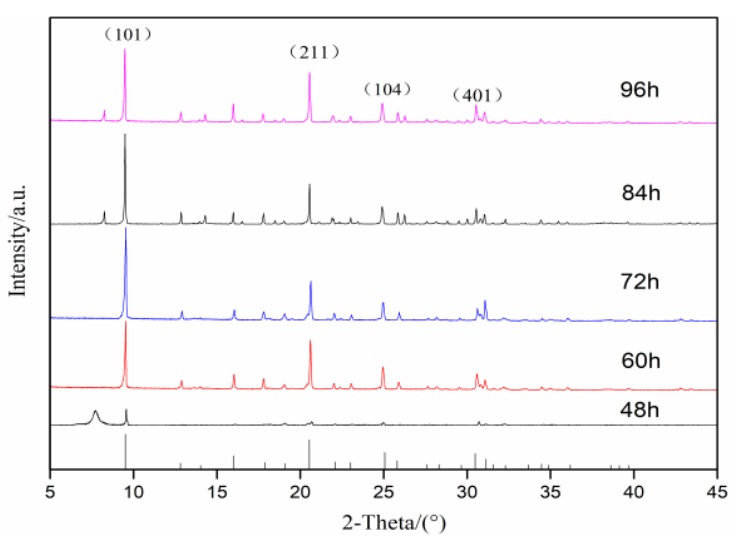

Figure 1. -X-ray diffraction (XRD) patterns of $\mathrm{FePO}_{4} @ \mathrm{SAPO}$ 34 at different crystallization times

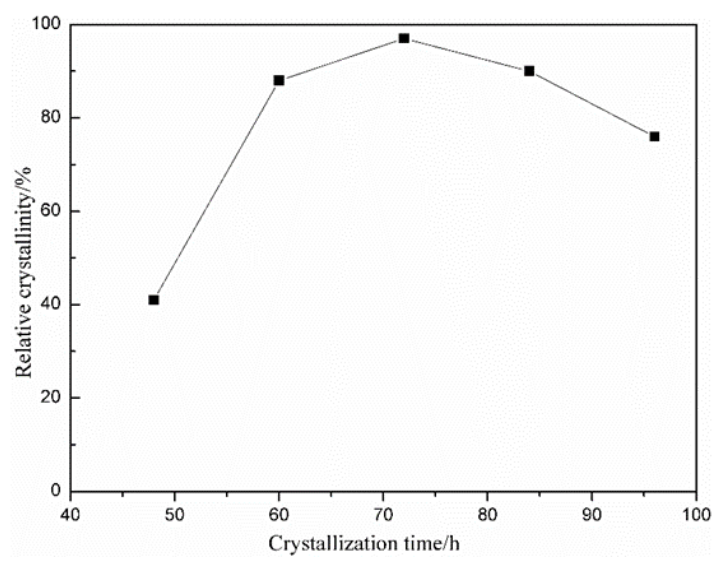

Figure 2.Crystallinity of $\mathrm{FePO}_{4} @ \mathrm{SAPO}-34$ at different crystallization times 


\subsection{SEM characterization}

The scanning electron microscopy (SEM) images showed, as the time increased from $48 \mathrm{~h}$ to $72 \mathrm{~h}$, the shape of the material became more and more complete. At $180{ }^{\circ} \mathrm{C}, \mathrm{t}=72 \mathrm{~h}, \mathrm{FePO} 4$ @SAPO-34 had a typical cubic small square structure of chabazite-based molecular sieves, indicating that the crystal growth was perfect [9-10]. When the time was gradually increased to 84$96 \mathrm{~h}$, the morphology of $\mathrm{FePO}_{4} @ \mathrm{SAPO}-34$ particles was destroyed, and some amorphous substances appeared in the synthesized product.

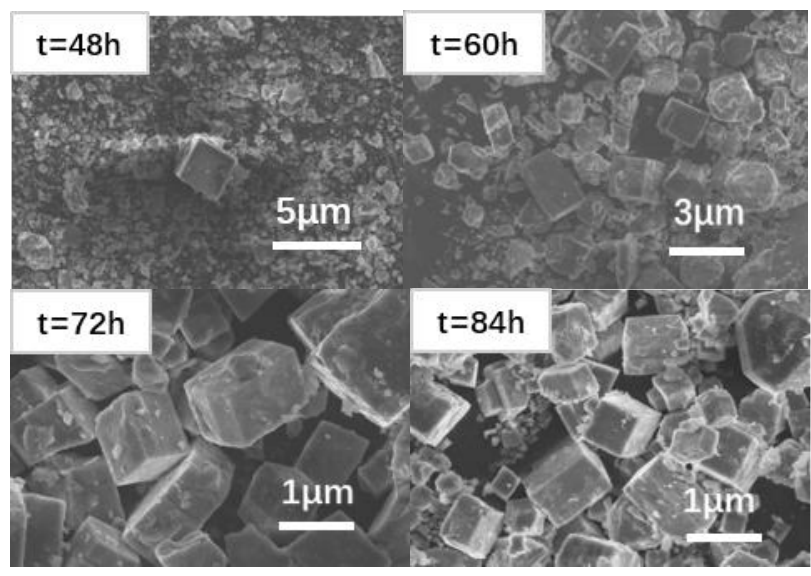

Figure 3. Scanning electron microscopy (SEM) images of FePO4@SAPO-34 at different crystallization times

\subsection{FT-IR and BET characterization}

FT-IR analysis was further carried out to verify FePO4@SAPO-34 in the reaction process. FT-IR spectrum was sensitive to the skeletal structure of the molecular sieve, and could better detect the molecular sieve crystallite structure. SAPO-34 has a periodic arrangement of $\mathrm{PO}^{2+}, \mathrm{AlO}_{4}$ and $\mathrm{SiO}_{4}$ tetrahedral framework structures. As shown in Fig 4, the spectrum of FePO4@SAPO-34 was basically consistent with the spectrum of SAPO-34. Peak at $2320 \mathrm{~cm}^{-1}$ could be ascribed to the bending vibration of $\mathrm{CO}_{2}$, while those at $730 \mathrm{~cm}^{-1}, 640 \mathrm{~cm}^{-1}, 575 \mathrm{~cm}^{-1}$ and $530 \mathrm{~cm}^{-1}$ could be assigned to the stretching vibration of OPO (O-Al-O), the double six-membered ring vibration peak and $\mathrm{PO}_{4}$. It may be due to moisture in the air during the test. When $\mathrm{FePO}_{4}$ was doped, since the electron energy of iron was smaller than the electron energy of silicon, the bonding strength of the sample was enhanced, and the corresponding frequency was increased[11-13].

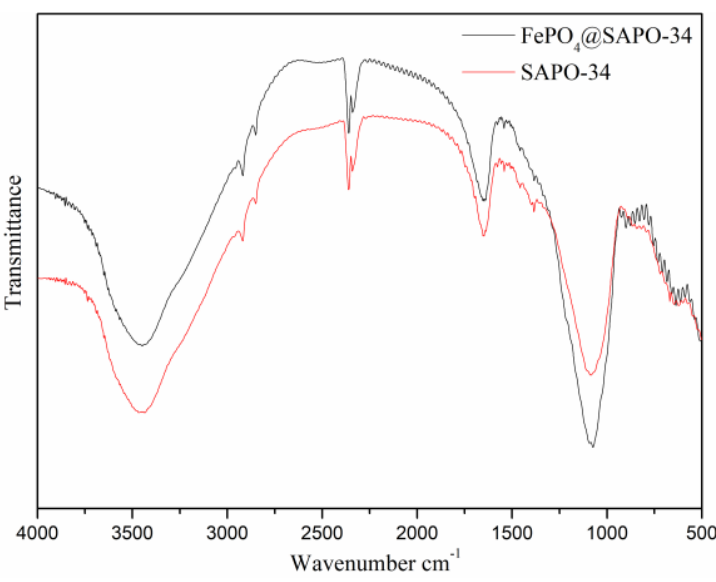

Figure 4. FT-IR image of $\mathrm{FePO}_{4} @ \mathrm{SAPO}-34$ and SAPO-34 samples

From Table 1 ,the specific surface of SAPO-34 was $391.2 \mathrm{~m}^{2} . \mathrm{g}-1$, and the specific surface of $\mathrm{FePO}_{4} @$ SAPO34 was $362.8 \mathrm{~m}^{2} . \mathrm{g}-1$. It had little difference and large specific surface area.

Table 1. BET of FePO4@SAPO-34 and SAPO-34

\begin{tabular}{|c|c|c|}
\hline Sampal & $\mathrm{FePO}_{4} @ S A P O-34$ & SAPO-34 \\
\hline $\begin{array}{c}\text { BET surface } \\
\text { area } /\left(\mathrm{m}^{2} \cdot \mathrm{g}^{-1}\right)\end{array}$ & 362.8 & 391.2 \\
\hline
\end{tabular}

\subsection{Adsorption of $\mathrm{pb}^{2+}$ experiments}

The removal effect of $\mathrm{Pb}^{2+}$ by SAPO-34 and FePO4@SAPO-34 materials has been shown in Fig.5. It can be seen that SAPO-34 has better absorbing capacity than FePO4@SAPO-34 in the first 45min, which was ascribed to the large specific surface area for pure SAPO-34 with a uniform pore structure[13-15]. However, the removal efficiency of two materials has been changed. The removal efficiency of FePO4@SAPO-34 exceeded to that of SAPO-34 due to the electrostatic effect of $\mathrm{FePO} 4$ in aqueous solution, further attracting cations which has been reported in other papers. Thus, the FePO4@SAPO-34 showed excellent adsorption performance in pollution removal.

On this basis, this experiment studied the effect of FePO4@SAPO-34 dosage on $\mathrm{Pb}^{2+}$ adsorption. As shown in Figure 6, as the amount of material increases, it can be seen that, the amount of $\mathrm{Pb}^{2+}$ adsorption increases. The increase was larger and then tended to be flat. The adsorption efficiency is calculated as follows:

$$
\eta=\frac{C_{0}-C_{i}}{C_{0}} \times 100 \%
$$

Where, $\mathrm{C}_{0}$ is original concentration; $\mathrm{C}_{\mathrm{i}}$ is concentration during dynamic adsorption equilibrium. $\eta$ is adsorption efficiency. When the addition amount was $0.2 \mathrm{~g}$, the adsorption rate was only $67.3 \%$. When the amount added was increased to 
$0.6 \mathrm{~g}$, the removal rate of $\mathrm{Pb}^{2+}$ was $89 \%$. When the amount added was $1 \mathrm{~g}$, the adsorption rate was $92 \%$. As the number of exchanged active particles increases, the amount of adsorption of metal ions becomes large[1416]. Therefore, the amount of adsorbent has a great influence on the adsorption effect.

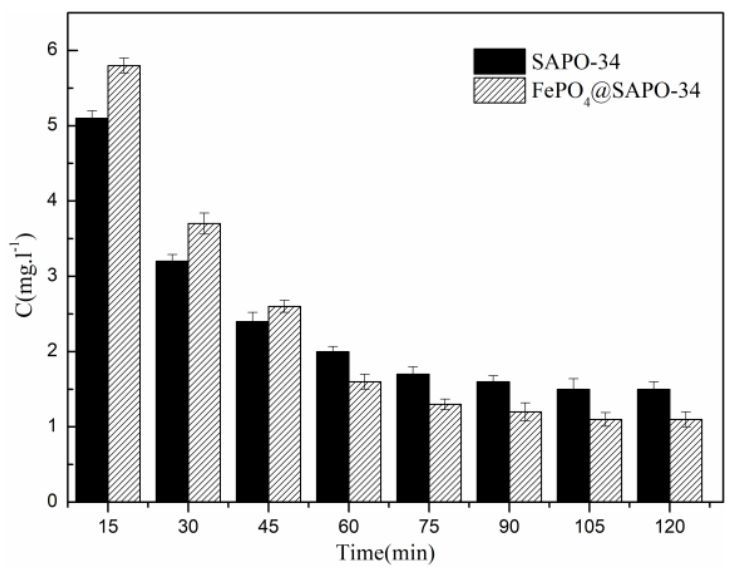

Figure 5. $\mathrm{Pb}^{2+}$ removal effect image of $\mathrm{FePO}_{4} @ \mathrm{SAPO}-34$

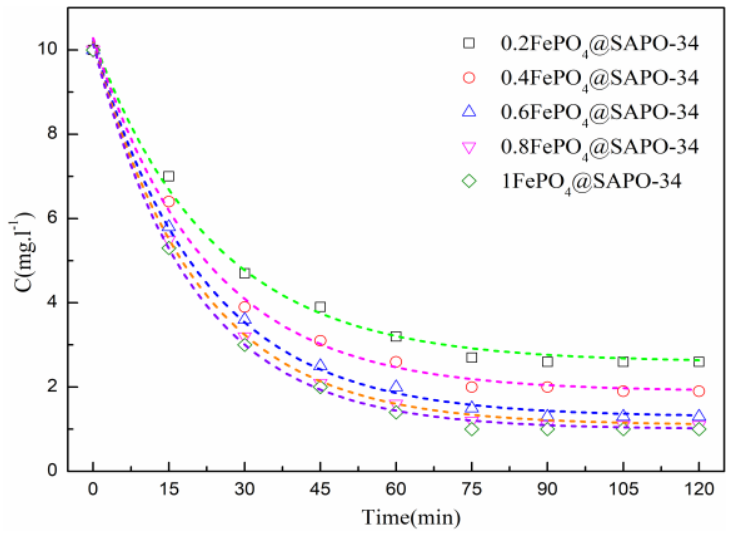

Figure 6. Adsorption effect diagram for different adsorbent dosages

\section{Conclusions}

In this study, an effective phosphate residue recovery method was proposed and be used to fabricate a FePO4@SAPO-34 adsorbent. Experiments were conducted to investigate the effect of different crystallization times on the crystal form of the adsorbent. The results showed that the synthesized sample crystal form was more complete at $72 \mathrm{~h}$. Experiments have found that new materials can effectively remove heavy metal ions from wastewater. Compared with SAPO-34, the new synthetic material passes through the electro-negative properties of $\mathrm{FePO}_{4}$, so it electrostatically adsorbs positively charged ions. And FePO4 provides a metal active center for SAPO-34. The synergy between SAPO-34 and FePO4@SAPO-34 improves the adsorption performance of new materials.

\section{Acknowledgement}

This work was supported by Shanghai Polytechnic University Graduate Program Foundation (No. EGD18YJ0005), Material Science and Engineering Key Subject of Shanghai Polytechnic University (No. XXKZD1601), and Gaoyuan Discipline of ShanghaiEnvironmental Science and Engineering (Resource Recycling Science and Engineering).

\section{References}

1. L. Wei, Y. Zhang, S. Chen, L. Zhu, X. Liu, L. Kong, L. Wang, J. Environ. Sci. 1-11 (2018)

2. Q. Sun, Z. Xie, J. Yu, National Science Review, 1-1 7 (2017)

3. L J. Wang, L L. Xie, H. Yuan, Q H. Li, Q Z. Li, AC A CHIMICA SINICA. 65, 170-172 (2007)

4. L J. Wang, H T. Zhao, Z X. Hao, H. Yuan, L L. Xie, Z. Tian, S W. Chen, J F. Wang, Q H. Li, ACTA CHI MICA SINICA. 65, 1929-1932 (2005)

5. L J. Wang, H T. Zhao, Z X. Hao, L L. Xu, L L. Xie, Z. Tian, H. Yuan, N C. Bing, Q H. Li, ACTA CHIMI CA SINICA. 66, 1317-1321 (2008)

6. W P. Zhu, Y P. Xue, Y. Li, S X. Tian, G. Yue, Mode rn Chemical Industry. 30 (2010)

7. L. Long, S. Xiao, Z Y. Li, S H. Qi, Y N. Men, W B. ao, Y N. Liu. BULLETIN OF THE CHINESE CER AMIC SOCIETY. 34 (2015)

8. A H. Xing, Q Y. Feng, X F. Zhang, J D. Jiang, IND USTRIAL CATALYSIS. 24 (2016)

9. M. Han, Dalian University of Technology. (2011)

10. S. Lin, Jilin University. (2011)

11. Y. Wang, Taiyuan University of Technology. (2008)

12. S Andonova, S Tamm, C Montreuil, et al. Applied Catalysis B Environmental, 180 (2016)

13. G. Feng, P. Cheng, W. Yan, M. Boronat, X. Li, J. $\mathrm{Su}$, J. Wang, Y. Li, A. Corma, R. Xu, J. Yu, 351 (2016)

14. M. Han, Dalian University of Technology. 2011

15. W J. Wang, C Z. Gen, Environmental Science and T echnology. 27 (2014)

16. Q. Sun, Z. Xie, J. Yu, National Science Review, 1-1 7 (2017) 